\title{
On the calibration of the COBE/IRAS dust emission reddening maps ${ }^{\star}$
}

\author{
C. M. Dutra ${ }^{1,2}$, A. V. Ahumada ${ }^{3}$, J. J. Clariá $^{3}$, E. Bica ${ }^{4}$, and B. Barbuy ${ }^{1}$ \\ 1 Universidade de São Paulo, Instituto de Astronomia, Geofísica e Ciências Atmosféricas, Rua do Matão 1226, \\ Cid. Universitária, São Paulo, 05508-900 SP, Brazil \\ 2 Universidade Estadual do Rio Grande do Sul, Rua Bompland 512, São Borja 97670-000, RS, Brazil \\ 3 Observatorio Astronómico de Córdoba, Laprida 854, 5000 Córdoba, Argentina \\ ${ }^{4}$ Universidade Federal do Rio Grande do Sul, Instituto de Fisica, CP 15051, Porto Alegre 91501-970, RS, Brazil
}

Received 10 January 2003 / Accepted 19 June 2003

\begin{abstract}
In this work we study the spectral properties (3600-6800 $\AA$ ) of the nuclear region of early-type galaxies at low $\left(|b|<25^{\circ}\right)$, intermediate (including surroundings of the Magellanic Clouds) and high (South Polar Cap) Galactic latitudes. We determine the $E(B-V)$ reddening values of the galaxies by matching their continuum distribution with respect to those of reddening-free spectral galaxy templates with similar stellar populations. We also compare the spectroscopic reddening value of each galaxy with that derived from $100 \mu \mathrm{m}$ dust emission $\left(E(B-V)_{\text {FIR }}\right)$ in its line of sight, and we find that there is agreement up to $E(B-V)=0.25$. Beyond this limit $E(B-V)_{\mathrm{FIR}}$ values are higher. Taking into account the data up to $E(B-V) \approx 0.7$, we derive a calibration factor of 0.016 between the spectroscopic $E(B-V)$ values and Schlegel et al.'s (1998) opacities. By combining this result with an $A_{K}$ extinction map built within ten degrees of the Galactic centre using Bulge giants as probes (Dutra et al. 2003), we extended the calibration of dust emission reddening maps to low Galactic latitudes down to $|b|=4^{\circ}$ and $E(B-V)=1.6\left(A_{V} \approx 5\right)$. According to this new calibration, a multiplicative factor of $\approx 0.75$ must be applied to the COBE/IRAS dust emission reddening maps.
\end{abstract}

Key words. ISM: dust, extinction - Galaxy: general - galaxies: ISM

\section{Introduction}

Galactic interstellar reddening is one of the observational limitations often challenging astronomers. Accurate maps of Galactic reddening are of crucial importance for a number of applications, such as measuring distances and mapping the peculiar velocity field in the nearby universe. Because of the patchy dust distribution in our Galaxy, it is very difficult to establish a general Galactic extinction law. However, various projects were undertaken in this direction. Sandage (1973) and de Vaucouleurs et al. (1976) modeled the interstellar extinction distribution as a function of the Galactic coordinates $(\ell, b)$. These general extinction laws were mainly used in extragalactic studies and have been adopted in the ShapleyAmes (Sandage \& Tamman 1981) and Second Reference (de Vaucouleurs et al. 1976) galaxy catalogues, respectively.

Send offprint requests to: C. M. Dutra,

e-mail: dutra@astro.iag.usp.br

* Based on observations made at Complejo Astronómico El Leoncito, which is operated under agreement between the Consejo Nacional de Investigaciones Científicas y Técnicas de la República Argentina and the National Universities of La Pata, Córdoba and San Juan, Argentina.
Burstein \& Heiles $(1978,1982)$ found relationships between galaxy counts, HI column densities and $E(B-V)$ reddening values. Additionally, they worked out reddening maps covering a great part of the sky, excepting the latitudes lower than $|b|<10^{\circ}$. Since the dust grains lie in the interstellar medium associated with the HI gas (Reach et al. 1998), Burstein \& Heiles' model reproduced the irregularities of the dust distribution more precisely. This is one of the reasons why Burstein \& Heiles maps have been widely used in the literature. More recently, Schlegel et al. (1998, hereafter SFD98) provided a new estimator of Galactic reddening by means of a full-sky $100 \mu \mathrm{m}$ IRAS/ISSA map, which was converted to dust column density by using a dust colour temperature map (17 K to $21 \mathrm{~K}$ ) derived from 100 and $240 \mu \mathrm{m}$ COBE/DIRBE dust emission maps. We will refer to these reddening maps from the IRAS/ISSA and COBE/DIRBE experiments by COBE/IRAS dust emission reddening maps, following Chen et al. (1999). The calibration of the relationship between the dust column density or opacity $\tau_{\text {FIR }}$ and SFD98's dust emission reddening $E(B-V)_{\text {FIR }}$ was made using a sample of early-type galaxies with an uncertainty of abou $16 \%$ in their reddening values, up to $E(B-V)=0.15$. The COBE/IRAS dust emission reddening maps cover the whole sky with a resolution of $6.1^{\prime}$. They show 
a good agreement with Burstein \& Heiles' maps at intermediate and high Galactic latitudes. However, SFD98 did not test their maps towards low Galactic latitudes and in the directions of the Magellanic Clouds. Given their characteristics, SFD98's maps supersede those of Burstein \& Heiles and different authors have tested the $E(B-V)_{\text {FIR }}$ values with independent reddening estimates derived from the stellar content of Galactic objects. Hudson (1999) compared the reddening predictions of these maps with the $E(B-V)$ values estimated for 50 distant globular clusters with $|b|>10^{\circ}$ and distances perpendicular to the Galactic plane $|Z|>3 \mathrm{kpc}$, as well as with those of 86 RR Lyrae stars from the sample of Burstein \& Heiles (1978). The reddening comparisons were carried out up to $E(B-V) \approx 0.30$ and resulted in reddening differences of $\delta E(B-V)=-0.008$ and -0.016 for the two samples, respectively.

On the other hand, Dutra \& Bica (2000) compared $E(B-V)$ reddening values from the stellar content of 103 old open clusters and 147 Galactic globular clusters with those derived from $E(B-V)_{\text {FIR }}$ maps. They obtained comparable reddening values between the two procedures for star clusters at $|b|>20^{\circ}$, in agreement with the fact that most of these clusters are located beyond the disk dust layer. However, Dutra \& Bica (2000) found significant differences for clusters at low Galactic latitudes located or projected in the disk dust layer. For low Galactic latitude star clusters, the differences are due to the background dust contribution, since the heated dust in the plane and towards the Galactic centre contributes only to the $E(B-V)_{\mathrm{FIR}}$ values. Considering 131 globular clusters with $|b|>2.5^{\circ}$ and $|Z|>100 \mathrm{pc}$ (assumed dust scale height), Chen et al. (1999) concluded that SFD98's reddening maps overestimated the visual absorption by a mean factor of 1.16. Arce \& Goodman (1999) analyzed the interstellar reddening in the Taurus dark cloud complex $\left(b \approx-15^{\circ}\right)$ by comparing $E(B-V)_{\text {FIR }}$ reddening values with those derived from four other methods and concluded that SFD98's reddening values overestimated the extinction by a factor of 1.3-1.5 in regions of smooth extinction with $A_{V}>0.5$. Dutra et al. (2002) built $A_{K}$ extinction maps using 2MASS data for two low extinction regions (windows) in the inner Bulge. The comparison of extinction values within a radius of one degree of window W359.4-3.1 (at $b=3.1^{\circ}$, and mean $E(B-V)=0.8$ ) indicated that the dust emission extinction values are overestimated by a factor of 1.45 with respect to those derived from 2MASS photometry. Also using 2MASS data, Dutra et al. (2003) built an $A_{K}$ extinction map within ten degrees of the Galactic centre, finding that for $3^{\circ}<|b|<5^{\circ}$, the discrepancy between reddening values derived from the dust emission and 2MASS photometry can be explained by a calibration factor of 1.31 .

Dutra et al. (2001, hereafter Paper I) obtained spectra from the nuclear region of galaxies behind and around the Magellanic Clouds in order to estimate the reddening in their lines of sight. They derived the reddening in each line-of-sight by matching the continuum distribution of the galaxy's spectrum with that of a reddening-free template with similar stellar population. For galaxies in the surroundings of the Magellanic Clouds, they concluded that the spectroscopic and dust emission reddening values agree well. They also detected the effect

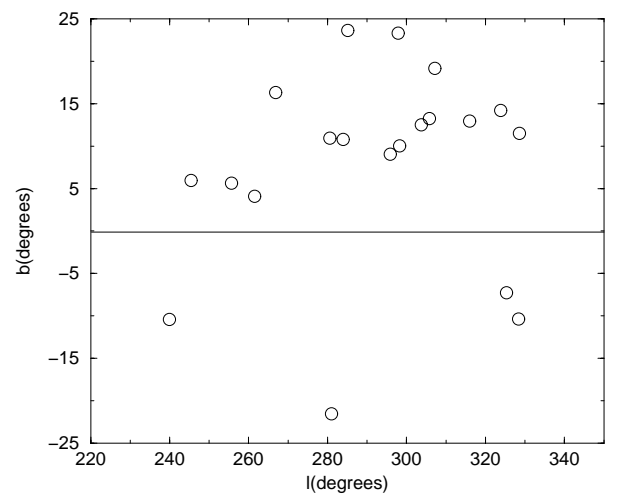

Fig. 1. Angular distribution of the observed sample of low Galactic latitude galaxies.

of the internal reddening of the Magellanic Clouds for galaxies behind them.

From the studies mentioned above, we can infer that the dust emission reddening maps - with their present reddeningdust emission calibration - need an additional calibration factor to reproduce reddening values higher than $E(B-V)=0.3$. In the current work, we use the spectroscopic method described in Paper I to derive independent foreground reddening estimates for early-type galaxies in lines of sight at low Galactic latitudes $\left(b<25^{\circ}\right)$, in order to compare these reddening values with those derived from dust emission. By using the present sample of galaxies, which provides reddening estimates for a wide sky coverage and by combining them to results cited above, we aim to extend the calibration of the dust emission reddening maps to regions with $|b|>4^{\circ}$ and more heavily reddened.

In Sect. 2 we present the sample of the observed early-type galaxies at low Galactic latitudes. In Sect. 3 we describe the observations and reductions, whereas in Sect. 4 we compare the present galaxy spectra with those of red stellar population templates obtained in Paper I to derive the reddening values. We also discuss some discrepancies appearing between the spectroscopic reddening values and those derived from the $100 \mu \mathrm{m}$ dust emission reddening maps. In Sect. 5 we provide a new reddening calibration for the COBE/IRAS dust emission reddening maps using the galaxy sample and extend it using literature data. In Sect. 6 we test the new calibration using the intrinsic $(B-V)_{0}$ colour $\times \mathrm{Mg}_{2}$ index relation for 311 galaxies. Concluding remarks are given in Sect. 7.

\section{The sample}

We used the LEDA database (http://leda.univlyon $1 . \mathrm{fr}$ ) as a starting point to select early-type galaxies with Galactic latitude $|b|<25^{\circ}$ and total blue magnitude $B_{\mathrm{T}}<15$. Figure 1 shows the angular distribution of the observed galaxies. The present sample and its properties are given in Table 1, which includes the following columns: (1) designation, (2) and (3) J2000 equatorial coordinates, (4) and (5) Galactic coordinates, (6) total blue magnitude $B_{\mathrm{T}}$, (7) exposure time, (8) radial velocity measurement, (9) LEDA and/or NED radial velocity, and (10) LEDA/NED morphological type. 
Table 1. The sample of observed galaxies.

\begin{tabular}{|c|c|c|c|c|c|c|c|c|c|}
\hline Object & $\begin{array}{c}\text { RA(2000) } \\
\text { h:m:s }\end{array}$ & $\begin{array}{c}\operatorname{Dec}(2000) \\
\circ: 1: 1,\end{array}$ & $\begin{array}{c}\ell \\
\left({ }^{\circ}\right)\end{array}$ & $\begin{array}{c}b \\
\left({ }^{\circ}\right)\end{array}$ & $B_{\mathrm{t}}$ & $\begin{array}{l}\text { Exp } \\
(s)\end{array}$ & $\begin{array}{c}V \\
\left(\mathrm{~km} \mathrm{~s}^{-1}\right)\end{array}$ & $\begin{array}{c}V_{\text {lit }} \\
\left(\mathrm{km} \mathrm{s}^{-1}\right)\end{array}$ & Type \\
\hline \multicolumn{10}{|c|}{ CASLEO $2.15 \mathrm{~m}$ sample } \\
\hline PGC 32955 & $10: 57: 48$ & $-47: 50: 36$ & 283.96 & 10.80 & 14.49 & $3 \times 1200$ & 5761 & - & E-S0 \\
\hline ESO 137-45 & $16: 51: 03$ & $-60: 48: 30$ & 328.34 & -10.39 & 13.19 & $3 \times 1200$ & 3450 & 3351 & $\mathrm{E}$ \\
\hline ESO 171-8 & $12: 04: 46$ & $-53: 10: 27$ & 295.88 & 9.06 & 14.19 & $3 \times 1200$ & 4544 & 4423 & $\mathrm{E}$ \\
\hline ESO 218-2 & $12: 21: 09$ & $-52: 35: 09$ & 298.27 & 10.01 & 13.66 & $2 \times 1200$ & 4520 & 4280 & $\mathrm{E}$ \\
\hline ESO 221-26 & $14: 08: 24$ & $-47: 58: 13$ & 316.01 & 12.94 & 12.10 & $2 \times 1200$ & 1517 & 2788 & $\mathrm{E}$ \\
\hline ESO 264-31 & $10: 40: 33$ & $-46: 11: 29$ & 280.53 & 10.91 & 13.91 & $3 \times 1200$ & 6605 & 6724 & $\mathrm{E}$ \\
\hline ESO 269-72 & $13: 13: 34$ & $-43: 31: 35$ & 307.18 & 19.16 & 13.98 & $3 \times 1200$ & 2912 & 3084 & $\mathrm{E}$ \\
\hline ESO 273-2 & $14: 46: 31$ & $-43: 57: 10$ & 323.87 & 14.19 & 14.19 & $2 \times 1200$ & 5200 & - & E-S0 \\
\hline ESO 274-6 & $15: 16: 10$ & $-44: 00: 50$ & 328.61 & 11.52 & 14.45 & $3 \times 1200$ & 4942 & 4458 & $\mathrm{E}$ \\
\hline ESO 314-2 & $08: 57: 29$ & $-39: 16: 09$ & 261.50 & 4.10 & 12.91 & 1200 & 1297 & 982 & $\mathrm{E}$ \\
\hline IC 3896 & $12: 56: 44$ & $-50: 20: 43$ & 303.80 & 12.52 & 12.18 & $2 \times 1200$ & 2148 & 2110 & $\mathrm{E}$ \\
\hline NGC 2663 & 08:45:08 & $-33: 47: 40$ & 255.67 & 5.65 & 11.99 & $2 \times 1200$ & 2243 & 2122 & $\mathrm{E}$ \\
\hline ESO 137-8 & $16: 15: 46$ & $-60: 55: 07$ & 325.34 & -7.28 & 13.21 & $3 \times 1200$ & 4110 & 3833 & $\mathrm{E}$ \\
\hline \multicolumn{10}{|c|}{ ESO $1.52 \mathrm{~m}$ sample } \\
\hline IC 2311 & $08: 18: 46$ & $-25: 22: 14$ & 245.44 & 5.95 & 12.50 & $2 \times 1800$ & 1960 & 1844 & $\mathrm{E}$ \\
\hline NGC 3087 & 09:59:09 & $-34: 13: 30$ & 266.89 & 16.32 & 12.46 & 1800 & 2680 & 2626 & $\mathrm{E}$ \\
\hline NGC 3706 & $11: 29: 45$ & $-36: 23: 27$ & 285.08 & 23.63 & 12.33 & $2 \times 900$ & 3100 & 2978 & E-S0 \\
\hline NGC 2325 & 07:02:40 & $-28: 41: 50$ & 239.96 & -10.41 & 12.30 & $2 \times 1800$ & 2050 & 2164 & $\mathrm{E}$ \\
\hline NGC 4976 & 13:08:38 & $-49: 30: 17$ & 305.80 & 13.27 & 10.96 & $2 \times 1200$ & 1600 & 1444 & $\mathrm{E}$ \\
\hline IC 3370 & $12: 27: 37$ & $-39: 20: 16$ & 297.92 & 23.30 & 12.03 & $2 \times 1800$ & 3070 & 2935 & E \\
\hline NGC 2434 & $07: 34: 52$ & $-69: 17: 02$ & 281.00 & -21.54 & 12.36 & $2 \times 1800$ & 1550 & 1400 & $\mathrm{E}$ \\
\hline
\end{tabular}

Table 2. Instrument and spectra general characteristics.

\begin{tabular}{|c|c|c|c|c|c|c|c|c|c|}
\hline Observatory/tel & spectrograph & $\begin{array}{l}\text { size CCD } \\
\text { (pixels) }\end{array}$ & $\begin{array}{c}\text { pixel size } \\
\left(\mu \mathrm{m}^{2}\right)\end{array}$ & $\begin{array}{c}\text { grating } \\
\left(\text { grooves } \mathrm{mm}^{-1} \text { ) }\right.\end{array}$ & $\begin{array}{c}\text { dispersion } \\
(\AA ̊ \text { Àixel })\end{array}$ & $\begin{array}{c}\text { spectral coverage } \\
(\AA)\end{array}$ & $\begin{array}{c}\text { width slit } \\
\left({ }^{\prime \prime}\right)\end{array}$ & $\begin{array}{l}\text { resolution } \\
(\AA)\end{array}$ & $\begin{array}{c}\text { lenght slit } \\
\text { (') }\end{array}$ \\
\hline ESO $1.52 \mathrm{~m}$ & $\mathrm{~B} \& \mathrm{C}$ & $2688 \times 512$ & $15 \times 15$ & 300 & 3.72 & $3600-10100$ & 3.5 & 18 & 4.1 \\
\hline CASLEO $2.15 \mathrm{~m}$ & $\mathrm{~B} \& \mathrm{C}$ & $1024 \times 1024$ & $24 \times 24$ & 300 & 3.43 & $3600-6800$ & 4.0 & 14 & 4.7 \\
\hline
\end{tabular}

\section{Observations and reductions}

The spectra were collected with the 1.52-m telescope at the European Southern Observatory (ESO, La Silla, Chile) on 11-14 January 2002 and 6-7 December 2002, and the 2.15-m telescope at the Complejo Astronómico El Leoncito (CASLEO, San Juan, Argentina) on 10-12 May 2002. In both telescopes we employed a CCD camera attached to a Boller \& Chivens spectrograph. At least two exposures of each object were taken in order to correct for cosmic rays. The exposure times are given in Table 1, while Table 2 summarizes the instrumental and general characteristics of the spectra obtained in the two Observatories. The standard stars EG21, LTT2415, and LTT3864 (Baldwin \& Stone 1984) were observed for flux calibrations. He-Ar lamp exposures were taken following that of the object or standard star for wavelength calibrations. The slit was set in the $\mathrm{E}-\mathrm{W}$ direction and its length projected on the chip (Table 2) provided a wide range of pixel rows for background subtractions.

The reductions were carried out using the IRAF package following standard procedures. The spectra were bias corrected, flat-fielded and extracted along the slit according to the dimensions of each galaxy's nuclear region. Typical extractions were $\approx 8-10^{\prime \prime}$. Afterwards, they were calibrated in wavelength and flux units using a He-Ar lamp and standard star spectra, respectively. Corrections for atmospheric extinction were applied, adopting the standard mean extinction curves from ESO/La Silla and CASLEO (Minniti et al. 1989).

Since the spectral resolution was chosen for stellar population purposes, it is not ideal for radial velocity measurements. We measured in any case the radial velocities using the absorption bands/lines centred at the $G$ band (4301 $\AA$ ), $\mathrm{H}_{\beta}$ (4861 $\AA$ ), Mg I (5175 $\AA$ ) and Na I (5890 $\AA$ ). The measured velocities and those from the LEDA and NED extragalactic databases are 


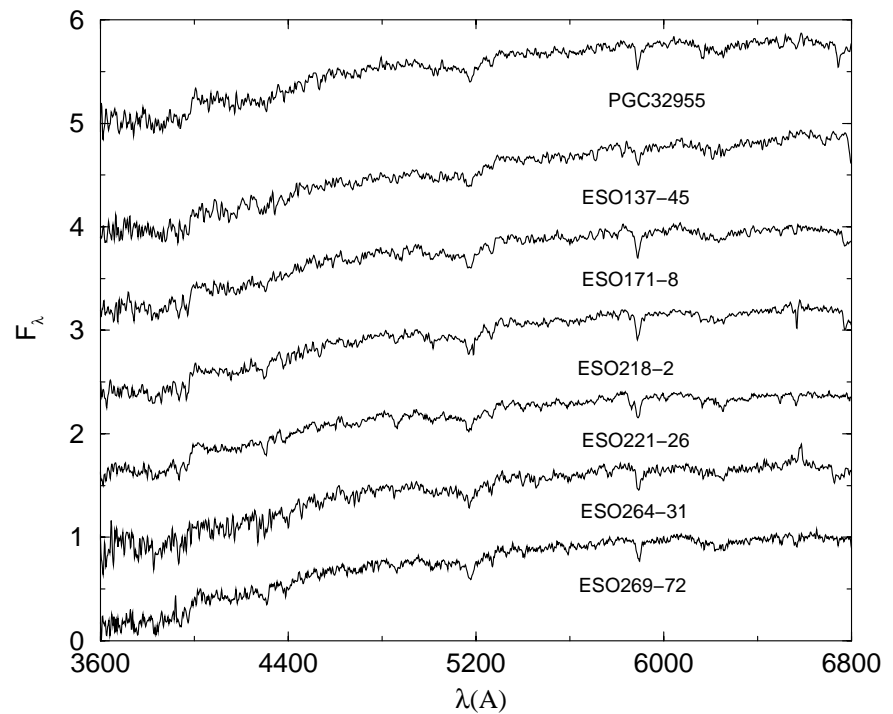

Fig. 2. Rest-frame spectra of galaxies observed at CASLEO. Spectra are in relative $F_{\lambda}$ units normalized at $5870 \AA$. Constants have been added to the spectra for the sake of clarity, except for the bottom one.

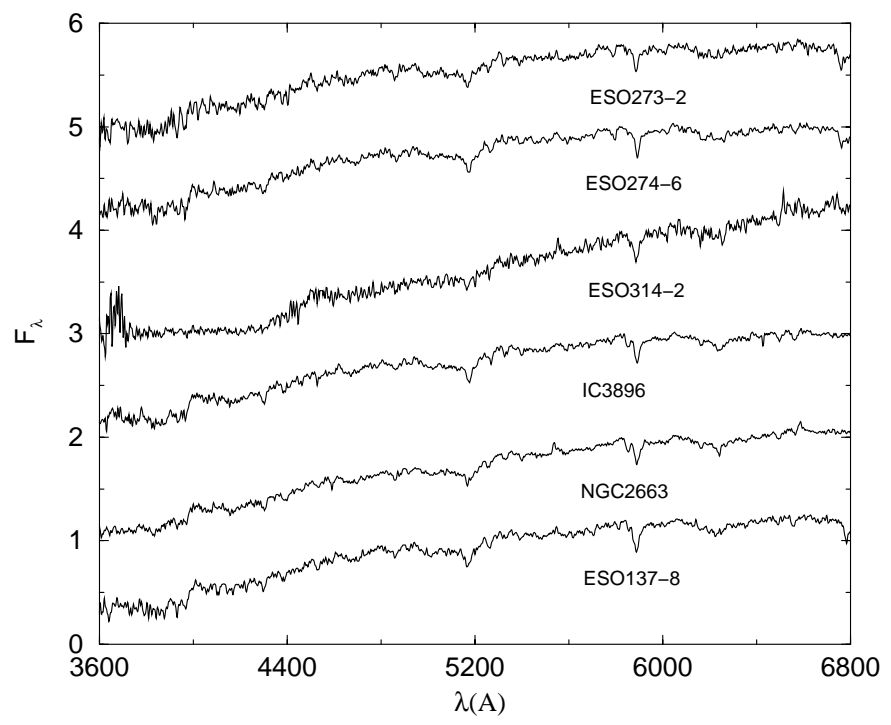

Fig. 3. Rest-frame spectra of additional galaxies observed at CASLEO. Units are as in Fig. 2.

consistent (Table 1). In one case, ESO 221-26, the LEDA velocity (Table 1) appears to be significantly different from that of present observation. The measured velocities were used to bring the galaxy spectra to the rest frame, which is necessary for the subsequent stellar population analysis.

Figures 2-4 show the resulting rest-frame flux calibrated spectra for the observed galaxies at CASLEO 2.15-m and ESO 1.52-m telescopes. Note that although the spectra taken with the ESO 1.52-m telescope yield a larger spectral coverage (Table 2), we adopted the range (3600-6800 $\AA$ ) for all the spectra.

\section{Reddening analysis}

All the observed spectra (Figs. 2-4) correspond to the nuclear region of early-type galaxies and, therefore, they are

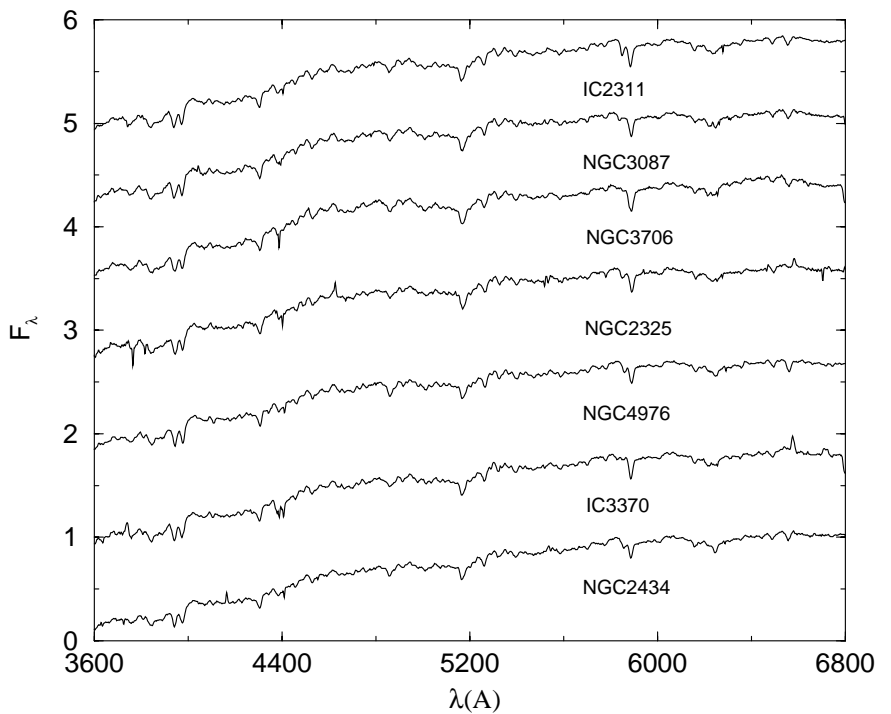

Fig. 4. Rest-frame spectra of galaxies observed at ESO (La Silla). Units are as in Fig. 2.

characterized by a red stellar population. Bica (1988) studied the stellar populations of the nuclear regions of early and latetype galaxies by means of their integrated spectra. Red stellar population galaxy nuclei spectra are ideal as Galactic reddening probes since the spectral distribution is essentially insensitive to age variations of the components and presents a small dependence on metallicity. He grouped the spectra in order to form high signal-to-noise red and blue stellar population templates, which are corrected for reddening following Sandage's law (1973). These templates represent the most frequent types of stellar populations found in normal galaxy nuclei.

In Paper I we defined new red stellar population templates, T1 and T23, based on those of Bica (1988) and new observations of early-type galaxies in the South Polar Cap. These templates are reddening-corrected and formed by galaxies with dust emission reddening $E(B-V)_{\mathrm{FIR}}<0.02$. We adopted the T1 and T23 templates in the present study to perform the match between the continuum distribution of these spectra and those from the observed galaxies for the purpose of estimating the reddening in their lines of sight.

Internal reddening in early type galaxies might be a source of uncertainty in the present method. Van Dokkum \& Franx (1995) found high opacities in cores of ellipticals in a scale of 1-2" using HST. Ferrari et al. (1999) showed the presence of internal dust in $75 \%$ of 22 observed elliptical galaxies. By integrating in larger areas (10-20") they derived a typical reddening value of $E(B-V)=0.01$, thus within uncertainties of the present determinations (typically $\epsilon_{E(B-V)}=0.02$, Paper I). Therefore the internal reddening variations in ellipticals are not expected to affect the present results significantly, since our apertures are relatively large (Sect. 3).

For the spectral comparisons, we need to determine which template has a stellar population which most closely resembles that of the observed galaxy. This estimation is made by comparing the equivalent widths (Ws) of both spectra. Then, we employ Ws of strong absorption features from the sample galaxies and templates' spectra. We use the 
Table 3. Equivalent widths for strong absorption features in the templates and individual galaxy spectra.

\begin{tabular}{|c|c|c|c|c|c|c|c|c|c|}
\hline Object & $K$ & $\mathrm{H}_{\delta}$ & $\mathrm{CN}$ & $G$ & $\mathrm{H}_{\gamma}$ & $\mathrm{H}_{\beta}$ & MgI & $\mathrm{NaI}$ & $\mathrm{H}_{\alpha}$ \\
\hline Windows & $3908-3952$ & $4082-4124$ & $4150-4214$ & $4284-4318$ & $4318-4364$ & $4846-4884$ & $5156-5196$ & $5880-5914$ & $6540-6586$ \\
\hline & \multicolumn{9}{|c|}{ Red Population Templates } \\
\hline $\mathrm{T} 1$ & 18.6 & 6.1 & 15.0 & 9.6 & 4.3 & 3.4 & 11.3 & 7.0 & 0.2 \\
\hline \multirow[t]{2}{*}{$\mathrm{T} 23$} & 17.1 & 4.3 & 10.2 & 9.2 & 4.4 & 3.5 & 8.7 & 4.3 & 0.8 \\
\hline & \multicolumn{9}{|c|}{ CASLEO $2.15 \mathrm{~m}$ sample } \\
\hline PGC 32955 & 20.4 & 4.7 & 18.6 & 11.7 & 9.7 & 1.1 & 9.7 & 5.8 & 2.2 \\
\hline ESO 137-45 & 22.5 & 1.6 & 11.1 & 8.9 & 7.7 & 3.9 & 10.2 & 5.1 & 1.0 \\
\hline ESO 171-8 & 19.1 & 5.0 & 15.3 & 8.9 & 7.1 & 3.8 & 10.6 & 7.1 & 1.4 \\
\hline ESO 218-2 & 19.0 & 6.9 & 16.4 & 10.7 & 5.3 & 3.7 & 11.8 & 6.2 & 2.5 \\
\hline ESO 221-26 & 18.4 & 6.8 & 13.1 & 9.3 & 5.6 & 4.9 & 9.0 & 5.5 & 2.3 \\
\hline ESO 264-31 & 17.6 & 5.1 & 13.1 & 11.2 & 7.2 & 3.7 & 9.8 & 6.1 & - \\
\hline ESO 269-72 & 20.1 & 6.3 & 14.9 & 10.4 & 5.9 & 3.8 & 10.1 & 4.6 & 2.7 \\
\hline ESO 273-2 & 16.9 & 8.2 & 16.6 & 9.0 & 4.8 & 3.6 & 9.5 & 5.3 & 1.3 \\
\hline ESO 274-6 & 20.0 & 6.9 & 15.8 & 10.1 & 5.8 & 3.6 & 10.9 & 6.6 & 1.4 \\
\hline ESO $314-2$ & 34.1 & 30.3 & 57.3 & 26.1 & 21.7 & 5.2 & 10.3 & 7.0 & 1.8 \\
\hline IC 3896 & 20.9 & 7.4 & 18.5 & 10.6 & 6.3 & 4.4 & 11.9 & 6.5 & 2.2 \\
\hline NGC 2663 & 17.5 & 5.9 & 18.5 & 10.3 & 7.6 & 4.9 & 10.7 & 6.7 & 0.4 \\
\hline \multirow[t]{2}{*}{ ESO 137-8 } & 22.3 & 9.4 & 18.0 & 10.1 & 7.4 & 3.8 & 11.4 & 7.2 & 2.5 \\
\hline & \multicolumn{9}{|c|}{ ESO $1.52 \mathrm{~m}$ sample } \\
\hline IC 2311 & 17.3 & 6.9 & 15.7 & 11.1 & 7.4 & 4.6 & 9.6 & 5.1 & 1.6 \\
\hline NGC 3087 & 16.7 & 6.6 & 15.3 & 11.6 & 7.2 & 4.2 & 9.7 & 4.8 & 1.6 \\
\hline NGC 3706 & 17.4 & 7.2 & 16.5 & 11.6 & 7.4 & 3.9 & 10.1 & 6.0 & 2.0 \\
\hline NGC 2325 & 17.2 & 5.1 & 14.1 & 10.4 & 6.3 & 3.6 & 9.7 & 5.1 & 0.1 \\
\hline NGC 4976 & 15.0 & 6.8 & 13.4 & 10.5 & 8.3 & 5.6 & 8.5 & 4.6 & 2.5 \\
\hline IC 3370 & 14.7 & 4.9 & 12.7 & 10.2 & 6.4 & 4.6 & 9.7 & 5.0 & - \\
\hline NGC 2434 & 15.8 & 5.5 & 11.5 & 10.8 & 7.2 & 4.7 & 9.0 & 4.4 & 1.7 \\
\hline
\end{tabular}

following metal features: K CaII (3933 ̊), CN (4182 ̊), $G$ band $(4301 \AA), \operatorname{MgI}(5175 \AA)$ and NaI $(5890 \AA)$, together with four Balmer lines: $\mathrm{H}_{\delta}(4101 \AA), \mathrm{H}_{\gamma}(4340 \AA), \mathrm{H}_{\beta}(4861 \AA)$ and $\mathrm{H}_{\alpha}(6563 \AA)$. Table 3 shows $W$ values for templates and individual galaxies measured with continuum tracings and feature windows following Bica \& Alloin (1986) and Bica et al. (1994). Typical $W$ errors are $\approx 5 \%$ and depend mostly on uncertainties in the continuum positioning.

For each galaxy the spectroscopic reddening value $E(B-$ $V)_{\text {SPEC }}$ was obtained by fitting the observed galaxy spectrum to that of the corresponding template with similar $W \mathrm{~s}$, by applying continuum corrections according to the Seaton's (1979) Galactic extinction law.

Figure 5 illustrates the reddening determination for NGC 4976 (upper panel) and NGC 2663 (lower panel), of stellar populations similar to those of the templates $\mathrm{T} 23$ and $\mathrm{T} 1$, respectively. The reddening effect in the observed spectra are important. Table 4 provides the associated template, as well as the spectroscopic and dust emission reddening value for each galaxy.

Figure 6 shows the comparison between these two reddening estimates using the present data and those from Paper I for
34 early-type galaxies at intermediate latitudes, surroundings of the Magellanic Clouds and South Polar Cap. A qualitative analysis shows that the two reddening estimates are consistent up to $E(B-V)<0.25$, but beyond this limit the two available points indicate higher $E(B-V)_{\text {FIR values. Figure } 6 \text { suggests }}$ a discrepancy between spectroscopic and higher dust emission reddening values, which has also been detected in other reddening comparisons in the literature, as mentioned in Sect. 1. The spectroscopic reddening determination method is a promising technique to understand this discrepancy at high reddening values regime with a homogeneous way of expanding the sample to fainter galaxies and extending the analysis towards the infrared domain.

\section{Recalibrating COBE/IRAS dust emission reddening maps.}

The transformation of opacity $\tau_{\text {FIR }}$ to a reddening $E(B-V)_{\text {FIR }}$ map carried out by SFD98 uses the correlation between the intrinsic $(B-V)_{0}$ colour of elliptical galaxies and their $\mathrm{Mg}_{2}$ index. The $\mathrm{Mg}_{2}$ index described by Faber et al. (1989) has a tight correlation with $(B-V)_{0}$, which in turn can be used to obtain 


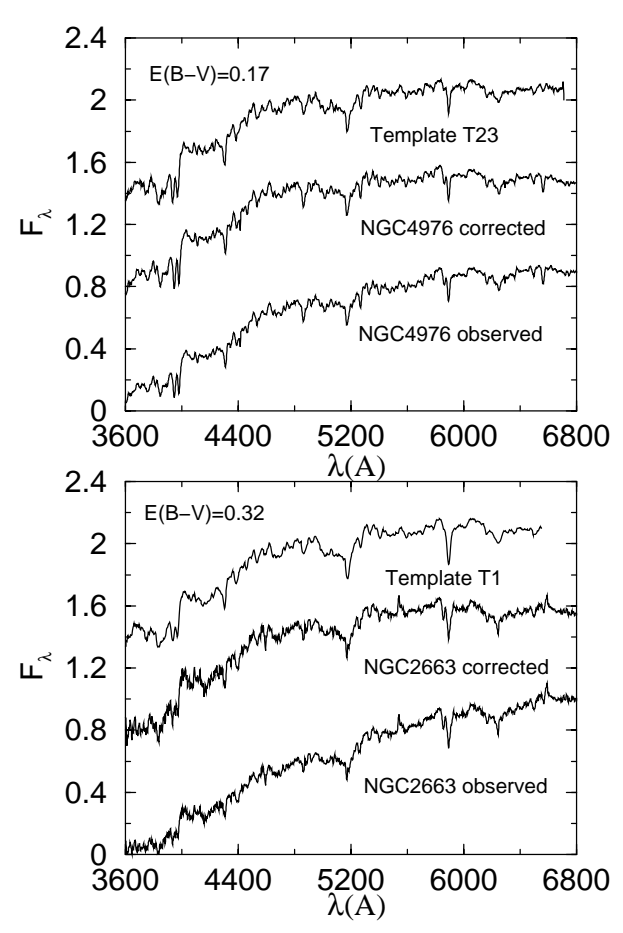

Fig. 5. Upper panel: reddening determination for NGC 4976. Lower panel: reddening determination for NGC 2663.

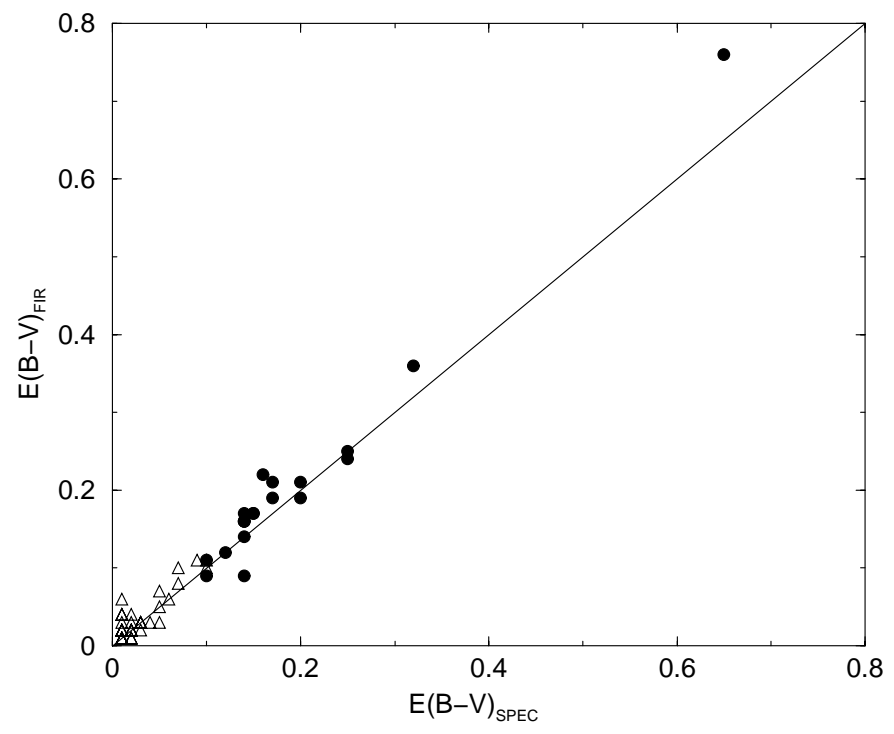

Fig. 6. Comparison between $E(B-V)$ reddening values derived from galaxy spectra and those from $100 \mu \mathrm{m}$ dust emission. The present data are indicated by filled circles, while Paper I's data are indicated by open triangles.

accurate reddening values. SFD98 used 389 elliptical galaxies from Faber et al. (1989) to compute a linear regression of reddening-corrected $(B-V)_{0}$ values against $\mathrm{Mg}_{2}$ with residuals $\delta(B-V)_{0}$. They computed the Spearman rank correlation coefficient of $\delta(B-V)_{0}$ versus the $E(B-V)_{\mathrm{FIR}}$ values, arguing that a good dust map will have no residual correlation. SFD98 obtained the following calibration factor:

$p=\frac{E(B-V)}{F_{100} X(T)}=0.0184 \pm 0.0014$,
Table 4. Spectroscopic and dust emission reddening values for observed galaxies from Bica (1988) and South Polar Cap samples.

\begin{tabular}{lccc}
\hline \hline Object & Template & $E(B-V)$ & $E(B-V)_{\mathrm{FIR}}$ \\
\hline PGC 32955 & $\mathrm{T} 1$ & 0.17 & 0.21 \\
ESO 137-45 & $\mathrm{T} 1$ & 0.25 & 0.24 \\
ESO 171-8 & $\mathrm{T} 1$ & 0.14 & 0.16 \\
ESO 218-2 & $\mathrm{T} 1$ & 0.15 & 0.17 \\
ESO 221-26 & $\mathrm{T} 23$ & 0.16 & 0.22 \\
ESO 264-31 & $\mathrm{T} 1$ & 0.14 & 0.16 \\
ESO 269-72 & $\mathrm{T} 1$ & 0.14 & 0.16 \\
ESO 273-2 & $\mathrm{T} 1$ & 0.14 & 0.17 \\
ESO 274-6 & $\mathrm{T} 1$ & 0.15 & 0.17 \\
ESO 314-2 & $\mathrm{T} 1$ & 0.65 & 0.76 \\
IC 3896 & $\mathrm{T} 1$ & 0.20 & 0.21 \\
NGC 2663 & $\mathrm{T} 1$ & 0.32 & 0.36 \\
ESO 137-8 & $\mathrm{T} 1$ & 0.20 & 0.19 \\
IC 2311 & $\mathrm{T} 1$ & 0.14 & 0.14 \\
NGC 3087 & $\mathrm{T} 1$ & 0.10 & 0.11 \\
NGC 3706 & $\mathrm{T} 1$ & 0.10 & 0.09 \\
NGC 2325 & $\mathrm{T} 1$ & 0.12 & 0.12 \\
NGC 4976 & $\mathrm{T} 23$ & 0.17 & 0.19 \\
IC 3370 & $\mathrm{T} 1$ & 0.14 & 0.09 \\
NGC 2434 & $\mathrm{T} 23$ & 0.25 & 0.25 \\
\hline
\end{tabular}

where $F_{100}$ is the $100 \mu \mathrm{m}$ IRAS flux expressed in $\mathrm{MJy} \mathrm{sr}^{-1}$ corrected from zodiacal emission, and $X(T)$ is the temperature correction factor to the fixed dust grain temperature $(18.2 \mathrm{~K})$ derived from the $100 / 240 \mu \mathrm{m}$ COBE/DIRBE maps. The product $F_{100} X(T)$ is defined as the FIR opacity $\tau_{\text {FIR. }}$.

Arce \& Goodman (1999) found that SFD98's reddening maps overestimated the reddening in the Taurus dark cloud complex by a factor of 1.3-1.5 in regions of high extinction $\left(A_{V}>0.5\right)$. They attributed this discrepancy to the fact that in the sample of 389 elliptical galaxies used to calculate a conversion from dust column density to $E(B-V), 90 \%$ of the galaxies have low-reddening $\left(E(B-V)_{\text {FIR }}<0.1\right)$ and very few have high-reddening $\left(E(B-V)_{\text {FIR }}>0.15\right)$ values. The lack of galaxies in high-reddening regions results in an inaccuracy in the conversion between dust column and reddening for lines of sight with $E(B-V)_{\mathrm{FIR}}>0.15$.

We use the present reddening estimates derived with the spectroscopic method to perform a new calibration of SFD98's opacities derived from $100 \mu \mathrm{m}$ emission and temperature correction maps. Figure 7's upper panel shows the comparison between spectroscopic reddening estimates and the corresponding SFD98's opacities. A linear regression to the data yields the following equation:

$E(B-V)=0.016 F_{100} X(T)+0.0008$

which includes a calibration factor slightly lower than that of Eq. (1). Figure 7's lower panel shows the comparison between SFD98's reddening estimates and those derived 

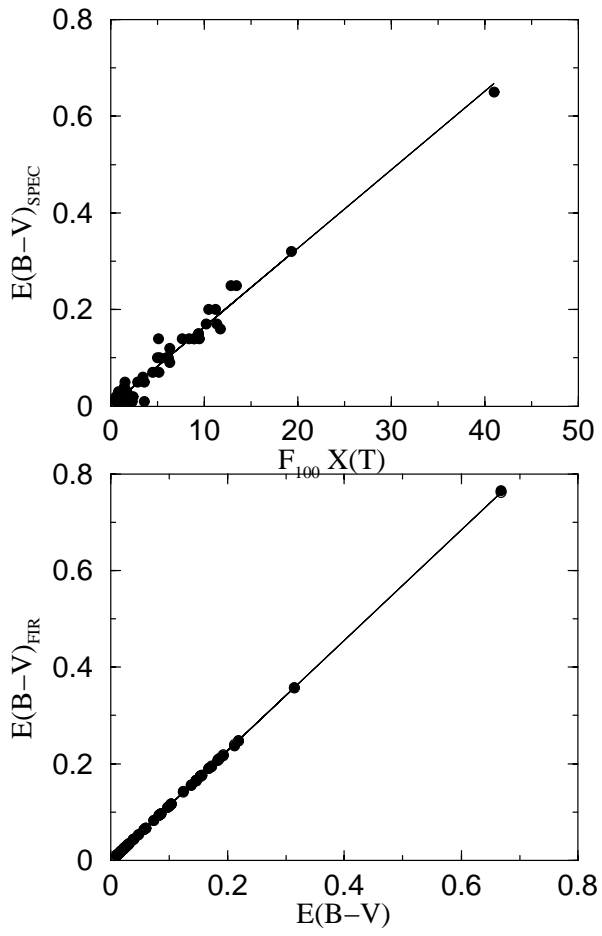

Fig. 7. Comparison of reddening determinations: i) upper panel, spectroscopic $E(B-V)_{\text {SPEC }}$ values versus SFD98's opacities $F_{100} X(T)$; ii) lower panel, SFD98's dust emission reddening $E(B-V)_{\mathrm{FIR}}$ values versus spectroscopic $E(B-V)$ colour excesses derived from Eq. (2).

from Eq. (2) using the opacities $\tau_{\text {FIR }}$. A linear regression to the data yields now:

$E(B-V)_{\mathrm{FIR}}=1.1412 E(B-V)-0.0015$

which indicates that $E(B-V)_{\text {FIR values overestimate reddening }}$ values by a factor of $\approx 1.14$ with respect to the present calibration for reddening values up to $E(B-V) \approx 0.7$. This result is in good agreement with that of Chen et al. (1999) who found that SFD98's reddening values overestimate the visual absorption by a factor of 1.16 for a sample of globular and open clusters with $|b|>2.5^{\circ}$, most of them with absorption $A_{V}<3$ $(E(B-V)<1)$.

In order to extend further the opacity-reddening calibration to higher $E(B-V)$ values, the $K$-band extinction $A_{K}$ map built by Dutra et al. (2003) within ten degrees of the Galactic center was used, together with the present reddening estimates.

Dutra et al. (2003) determined the $A_{K}$ extinction from $J H K_{\mathrm{s}}$ infrared 2MASS photometry of Bulge red giants, fitting the upper giant branch of $\left(K_{\mathrm{s}}, J-K_{\mathrm{s}}\right)$ colourmagnitude diagrams to a dereddened upper giant branch mean locus. These Bulge/Disk directions were divided into cells and about 6 million stars were used as reddening probes. Adopting $R_{V}=A_{V} / E(B-V)=3.1$ and $A_{K} / A_{V}=0.112$ (Cardelli et al. 1989), we transformed $A_{K}$ estimates to $E(B-V)$ ones for 3026 cells with $4 \times 4 \operatorname{arcmin}^{2}$ in size, located at Galactic coordinates $|\ell|<5^{\circ}$ and $4^{\circ}<|b|<5^{\circ}$, in a region where the dust background contribution to the $100 \mu \mathrm{m}$ flux (less than $5 \%$ ) is minimized and $A_{K}>2.5 \sigma_{i}$, where $\sigma_{i}$ is the uncertainty in the $A_{K}$ determination. The latter constraint is to warrant accuracy for the $A_{K}$ estimates. Figure 8 shows for the galaxy sample

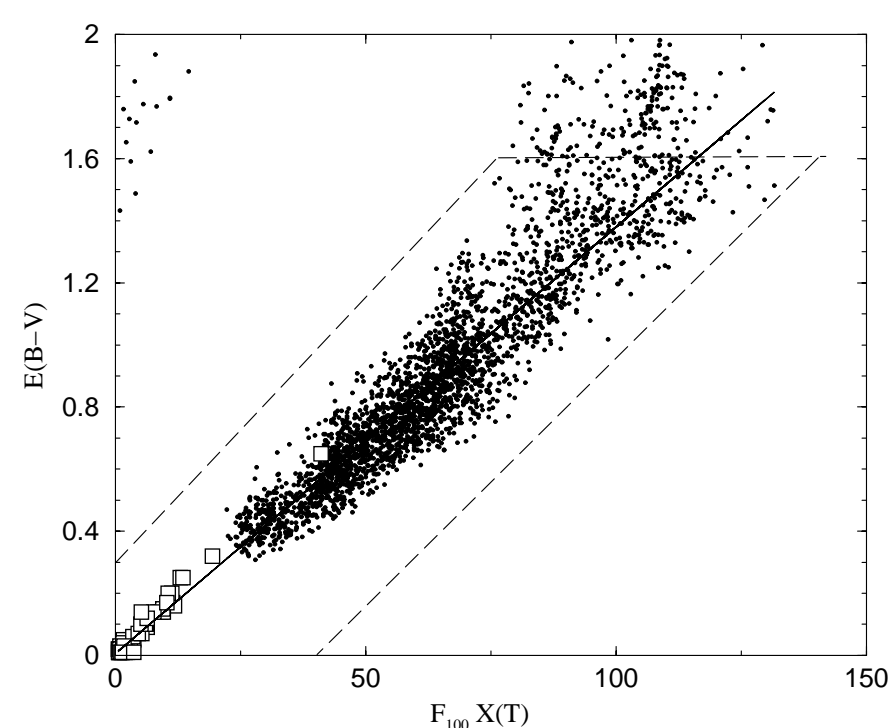

Fig. 8. Independent reddening values $E(B-V)$ versus SFD98's opacities $F_{100} X(T)$ for galaxy sample (open squares) and 3026 Bulge direction (filled circles).

and 3026 Bulge directions the comparison between independent reddening estimates and the SFD98's opacities. A linear regression fit within the polygon region (Fig. 8) yields the following new calibration:

$E(B-V)_{\mathrm{CAL}}=0.0137 F_{100} X(T)+0.0064$,

which extends up to $E(B-V)=1.6\left(\right.$ or $A_{V} \approx 5$ ).

Figure 9's panels (a) and (b) show for the galaxy sample and 3026 Bulge directions the comparisons between independent reddening estimates and those derived from SFD98 $E(B-V)_{\mathrm{FIR}}$ and $E(B-V)_{\mathrm{CAL}}$, respectively. In panel (a), the $E(B-V)_{\mathrm{FIR}}$ values appear to be systematically larger than the $E(B-V)$ ones, while panel (b) shows a good agreement between $E(B-V)_{\mathrm{CAL}}$ and $E(B-V)$, except for the points having $E(B-V)_{\mathrm{CAL}}<0.4$ and $1.4<E(B-V)<2.7$. The latter points correspond to those with $E(B-V)_{\mathrm{FIR}}>2.4$ and the same $E(B-V)$ range. In addition, Eq. (4) is not precise for higher reddening values of $E(B-V)>1.6$, where the slope of the $E(B-V)_{\mathrm{CAL}} \times F_{100} X(T)$ correlation has an abrupt variation (Fig. 8). In Fig. 9's panel (c) we present histograms of the relative differences $\chi_{\mathrm{CAL}}$ and $\chi_{\mathrm{FIR}}$, between $E(B-V)_{\mathrm{CAL}}$ and independent $E(B-V)$ measurements and $E(B-V)_{\text {FIR }}$ and $E(B-V)$ values, respectively. The relative differences $\chi_{\mathrm{CAL}}$ and $\chi_{\text {FIR }}$ are defined as:

$\left\{\begin{array}{l}\chi_{\mathrm{CAL}}=100 \times \frac{\sqrt{\left(E(B-V)_{\mathrm{CAL}}-E(B-V)\right)^{2}}}{E(B-V)} \\ \chi_{\mathrm{FIR}}=100 \times \frac{\sqrt{\left(E(B-V)_{\mathrm{FIR}}-E(B-V)\right)^{2}}}{E(B-V)} .\end{array}\right.$

As shown in Fig. 9's panel (c), 90\% of the points in the new calibration have a relative difference $\chi_{\mathrm{CAL}}<20 \%$, while the $\chi_{\text {FIR }}$ distribution has a peak at $\approx 35 \%$. This result shows that the present calibration proves to be an improvement in the studied reddening range. A comparison between present and SFD98's reddening estimates is shown in Fig. 9's panel (d). A linear 

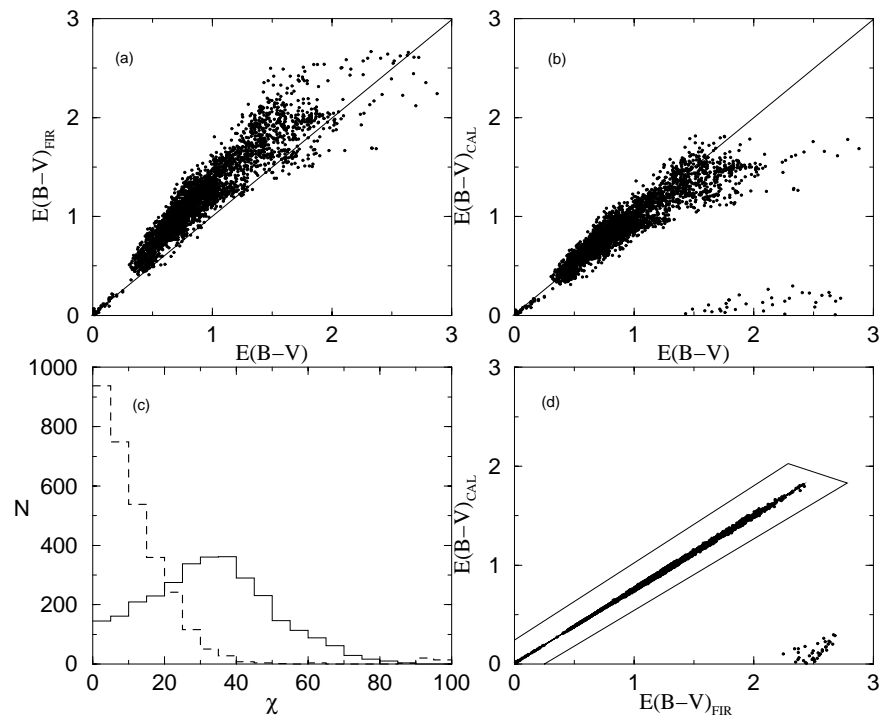

Fig. 9. Panels: a) $E(B-V)_{\mathrm{FIR}}$ versus $E(B-V)$, b) $E(B-V)_{\mathrm{CAL}}$ versus $E(B-V)$, c) histograms of the relative differences $\chi_{\mathrm{CAL}}$ (dashed line) and $\chi_{\mathrm{FIR}}$ (solid line), d) $E(B-V)_{\mathrm{CAL}}$ versus $E(B-V)_{\mathrm{FIR}}$.

regression fit within the polygon region (3044 points) leads to the following relation:

$$
E(B-V)_{\mathrm{CAL}}=0.7480 E(B-V)_{\mathrm{FIR}}+0.0056 .
$$

Equation (6) indicates that besides better reproducing the present data, our calibration could additionally provide good agreement with independent reddening measurements made by Arce \& Goodman (1999) in the Taurus dark cloud.

\section{Testing the FIR opacity-reddening calibration}

Hudson (1999) examined the large-scale systematic errors, which vary as a function of position on the sky, in Burstein \& Heiles and SFD98 reddening maps. He determined for 311 early-type galaxies a relation $\mathrm{Mg}_{2}$ index and intrinsic $(B-V)_{0}$ colour. We used the data by Hudson (1999) available at CDS to study the correlation between the $\mathrm{Mg}_{2}$ index and $(B-V)_{0}$ colour; for such, we dereddened the observed $(B-V)$ colour using SFD98's reddening estimates and those from the present calibration. Figure 10 shows in panels (a) and (c) the $(B-V)_{0}-\mathrm{Mg}_{2}$ relation, once the observed $(B-V)$ colours were corrected for reddening using SFD98's $E(B-V)_{\mathrm{FIR}}$ and the present calibration $E(B-V)_{\mathrm{CAL}}$, respectively. Panels (b) and (d) show the residuals from a linear fit to the $(B-V)_{0}-\mathrm{Mg}_{2}$ as a function of the respective reddening estimates. Comparing the residual plots, we note that for the same sample, $E(B-V)_{\mathrm{FIR}}$ values are higher than $E(B-V)_{\mathrm{CAL}}$ ones. The residual plot from the linear fit in (c) as a function of $E(B-V)_{\mathrm{CAL}}$ shows a more symmetric distribution than that

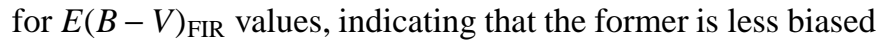
than the latter, mainly for higher reddening values. Blakeslee et al. (2001) performed the same test for 200 galaxies using a relation between the intrinsic $(V-I)_{0}$ colour and the $\mathrm{Mg}_{2}$ index. They concluded that SFD98 reddening values overestimate by $5-10 \%$ the interstellar reddening in the line-of-sight
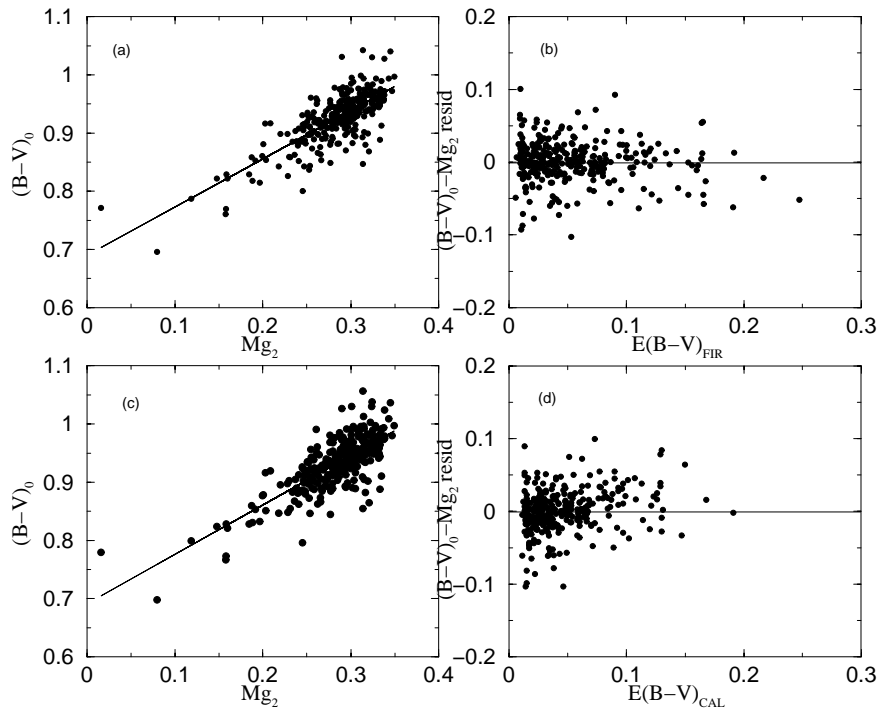

Fig. 10. Panels: a) $(B-V)_{0}$ versus $\mathrm{Mg}_{2}$ with $(B-V)_{0}$ corrected by $E(B-V)_{\text {FIR }}$ values $\left((B-V)_{0}=0.823 \mathrm{Mg}_{2}+0.689\right)$, b) residuals from the linear fit in a) as a function of $\left.E(B-V)_{\mathrm{FIR}}, \mathbf{c}\right)(B-V)_{0}$ versus $\mathrm{Mg}_{2}$ with $(B-V)_{0}$ corrected by $E(B-V)_{\mathrm{CAL}}$ values $\left((B-V)_{0}=\right.$ $\left.0.850 \mathrm{Mg}_{2}+0.691\right)$, d) residuals from the linear fit in $\left.\mathbf{c}\right)$ as a function of $E(B-V)_{\mathrm{CAL}}$.

of the galaxies with $E(B-V)>0.2$. This test is consistent with the present calibration.

\section{Concluding remarks}

We obtained spectra in the range (3600-6800 $\AA$ ) for the nuclear region of 20 early-type galaxies at low Galactic latitudes $\left(|b|<25^{\circ}\right)$ and total blue magnitude $B_{\mathrm{T}}<15$ using the Boller \& Chivens spectrograph at the CASLEO 2.15-m and ESO 1.52-m telescopes. Two of these galaxies have their radial velocities estimated for the first time.

We derived spectroscopically the line-of-sight reddening of these galaxies. The reddening in each line-of-sight was determined from the comparison of continuum distribution of the galaxy's spectrum with that of a reddening-free template with comparable stellar population according to the equivalent widths of its absorption features.

We compared the spectroscopic reddening estimates with those derived from the $100 \mu \mathrm{m}$ dust emission $\left(E(B-V)_{\mathrm{FIR}}\right)$ obtained by Schlegel et al. (1998) for the lines-of-sight of 54 galaxies, 20 of them from the present work and the remaining 34 from Paper I. The comparison reached reddening values up to $E(B-V)=0.65$ and indicates that for $E(B-V)>$ 0.25 the dust emission reddening estimates are higher than the spectroscopic reddening values, in agreement with other reddening comparisons carried out using different objects. The spectroscopic method proves to be useful for reddening determinations in the line-of-sight of low Galactic latitude galaxies and, therefore, it can be applied using larger telescopes to derive the interstellar reddening in the directions of fainter galaxies or of recently catalogued 2MASS galaxies using the infrared domain. The present and future reddening estimates 
using this method should constitute important observational constraints to new Galactic extinction models.

We used the present spectroscopic reddening estimates (up to $E(B-V) \approx 0.7$ ) and the SFD98's opacities (derived from $100 \mu \mathrm{m}$ emission and temperature correction maps) to recalibrate their dust emission reddening maps. We obtained a calibration factor of 0.016 between the spectroscopic $E(B-V)$ values and SFD98's opacities, which is a factor 0.88 lower than that obtained by SFD98. Using the $A_{K}$ extinction map recently built by Dutra et al. (2003) within ten degrees of the Galactic centre, we extended the calibration of dust emission reddening maps to low Galactic latitudes down to $|b|>4^{\circ}$ and reddening values of $E(B-V)=1.6\left(A_{V} \approx 5\right)$. According to the new calibration, a multiplicative factor of $\approx 0.75$ must be applied to the COBE/IRAS dust emission reddening maps in those regions.

Acknowledgements. We thank the CASLEO staff for hospitality and support during the observing run. The authors acknowledge use of the $C C D$ and data acquisition system supported under U.S. National Science Foundation grant AST-90-15827 to R. M. Rich. We have made use of the LEDA database, and the NASA/IPAC Extragalactic Database (NED), which is operated by the Jet Propulsion Laboratory, California Institute of Technology, under contract with the National Aeronautics and Space Administration. We employed an electronic table from CDS/Simbad (Strasbourg) and Digitized Sky Survey images from the Space Telescope Science Institute (U.S. Government grant NAG W-2166) obtained using the extraction tool from CADC (Canada). This work was partially supported by the Brazilian institutions FAPESP, CNPq and FINEP, the Argentine institutions CONICET, Agencia Córdoba Ciencia, ANPCyT and SECYT (UNC), and the VITAE and Antorchas foundations. CMD acknowledges FAPESP for a post-doc fellowship (proc. 2000/11864-6). We acknowledge FAPESP grant 1998/10138-8.

\section{References}

Arce, H. G., \& Goodman, A. A. 1999, ApJ, 512, L135

Baldwin, J. A., \& Stone, R. P. S. 1984, MNRAS, 206, 241

Bica, E. 1988, A\&A, 195, 76

Bica, E., \& Alloin, D. 1986, A\&A, 162, 21

Bica, E., Alloin, D., \& Schmitt, H. 1994, A\&A, 283, 805

Blakeslee, J. P., Lucey, J. R., Barris, B. J., et al. 2001, MNRAS, 327, 1004

Burstein, D., \& Heiles, C. 1978, ApJ, 225, 40

Burstein, D., \& Heiles, C. 1982, AJ, 87, 1165

Cardelli, J. A., Clayton, G. C., \& Mathis, J. S. 1989, ApJ, 345, 245

Chen, B., Figueras, F., Torra, J., et al. 1999, A\&A, 352, 459

de Vaucouleurs, G., de Vaucouleurs, A., \& Corwin, H. G. 1976, Second Reference Catalogue of Bright Galaxies (University of Texas Press) (RC2)

Dutra, C. M., \& Bica, E. 2000, A\&A, 359, 347

Dutra, C. M., Bica, E., Clariá, J. J., et al. 2001, A\&A, 371, 895 (Paper I)

Dutra, C. M., Santiago, B. X., \& Bica, E. 2002, A\&A, 381, 219

Dutra, C. M., Santiago, B. X., Bica, E., \& Barbuy, E. 2003, MNRAS, 338,253

Faber, S. M., Wegner, G., Burstein, D., et al. 1989, ApJS, 69, 763

Ferrari, F., Pastoriza, M. G., Macchetto, F., \& Caon, N. 1999, A\&AS, 138,269

Hudson, M. J. 1999, PASP, 111, 57

Minniti, D., Clariá, J. J., \& Gómez, M. 1989, Ap\&SS, 158, 9

Reach, W. T., Wall, W. F. \& Odegard, N. 1998, ApJ, 507, 507

Sandage, A. 1973, ApJ, 183, 711

Sandage, A., \& Tamman, G. 1981, A Revised Shapley-Ames Catalog of Bright Galaxies (Carnegie Inst. of Washington Publ.), 635

Schlegel, D. J., Finkbeiner, D. P., \& Davis, M. 1998, ApJ, 500, 525

Seaton, M. J. 1979, MNRAS, 187, 73

van Dokkum, P. G., \& Franx, M. 1995, AJ, 110, 2027 